\section{L’immunothérapie des cancers couronnée avec l'attribution du prix Nobel de Physiologie ou Médecine à James Allison et Tasuku Honjo} Jean-Luc Teillaud

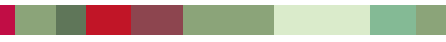

Le Docteur James Allison et le Professeur Tasuku Honjo ont reçu le prix Nobel de Physiologie ou Médecine en octobre 2018 pour «leur découverte du traitement du cancer par inhibition de la régulation immunitaire négative ». Dans ses attendus, l'Assemblée Nobel rappelle que James Allison a été parmi les premiers chercheurs à montrer que la molécule CTLA-4 (cytotoxic T lymphocyteassociated protein 4, ou CD152) - découverte de nombreuses années auparavant par Pierre Golstein au Centre d'immunologie de Marseille-Luminy (CIML) - agissait comme un frein sur les lymphocytes T engagés dans une réponse immunitaire dans son laboratoire de l'université de Californie, à Berkeley. Alors que ses collègues ciblaient cette molécule pour traiter des maladies auto-immunes, il a étudié les conséquences du blocage de CTLA-4 avec un anticorps antagoniste, en faisant l'hypothèse que cela permettait de libérer la réponse immunitaire contre les cellules tumorales, ce qu'il a pu démontrer en 1996 dans son laboratoire (Cancer Research Laboratory) à l'université de Californie à Berkeley. L'Assemblée Nobel rappelle que, malgré le peu d'intérêt qu'avait alors porté l'industrie pharmaceutique à cette découverte, James Allison a poursuivi ses efforts pour développer une stratégie thérapeutique utilisant un anticorps anti-CTLA-4 pour traiter des patients cancéreux. Ses efforts ont permis d'aboutir en 2010 à la mise en évidence d'un effet thérapeutique majeur de l'utilisation d'un tel anticorps chez des patients présentant un mélanome métastatique. ${ }^{1}$

Dans la suite de ses attendus, l'Assemblée Nobel rappelle que Tasuku Honjo a découvert PD-1 (programmed cell death-1), également exprimé sur les lymphocytes T, en 1992. Elle souligne également que celui-ci a, au cours des années suivantes, méthodiquement exploré les fonctions de cette molécule dans son laboratoire de l'université de Kyoto. Les résultats qu'il a alors obtenus ont montré que PD-l fonctionnait également comme un frein sur les lymphocytes $T$, mais par un mécanisme différent de celui de CTLA-4. Des expériences chez des souris porteuses de

Vignette (Photos III. Niklas Elmehed. (๐) Nobel Media)

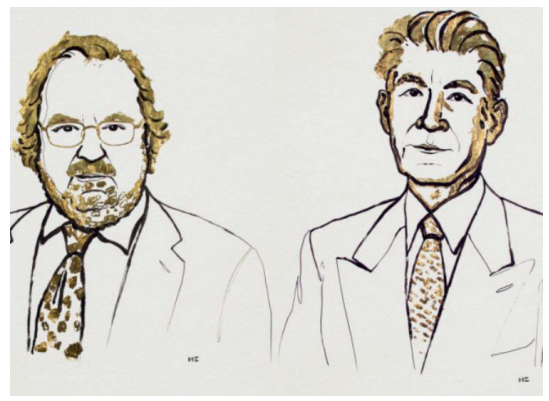

Rédacteur en chef de médecine/sciences jean-luc.teillaud@inserm.fr

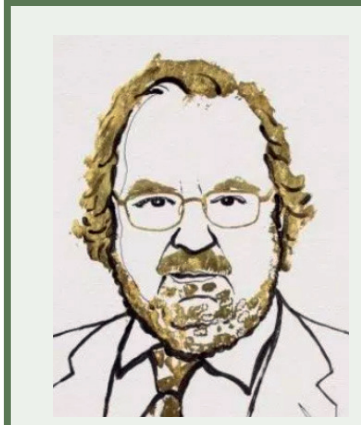

James P. Allison est né en 1948 à Alice au Texas. Il a obtenu son doctorat (PhD) en 1973 à l'université du Texas à Austin et a effectué son stage postdoctoral à la Scripps Clinic and Research Foundation à La Jolla en Californie de 1974 à 1977. II a été ensuite nommé professeur au «System Cancer Center » de l'université du Texas à Smithville de 1977 à 1984 et a rejoint l'université de Californie à Berkeley en 1985 où il a développé ses travaux de recherche fondamentale sur CTLA-4 jusqu'en

2004 et a effectué ses expériences montrant que le blocage de CTLA-4 par un anticorps antagoniste permettait le développement d'une réponse immunitaire accrue aboutissant au rejet de la tumeur, dans un modèle murin [1]. II est alors parti poursuivre ses travaux au Memorial SloanKettering Cancer Center (MSKCC) à New York de 2004 à 2012 et est finalement revenu en 2012 au Texas, au MD Anderson Cancer Center de l'université du Texas à Houston, où il est directeur de la plateforme d'immunothérapie du «Parker Institute for Cancer Immunotherapy », professeur d'immunologie et président du département d'immunologie (https://www. parkerici.org/person/james-p-allison-phd/). James Allison est également membre de l'Académie des sciences des États-Unis.

tumeurs qu'il a effectuées dans son laboratoire ont alors montré l'intérêt de bloquer l'interaction de PD-l avec son ligand PD-Ll en utilisant des anticorps antagonistes. II s'en est suivi un développement clinique qui a abouti en 2012 à la démonstration d'une claire efficacité du traitement par anticorps chez des patients ayant différents types de tumeurs, avec des rémissions à long terme, voire une guérison chez certains d'entre eux. 


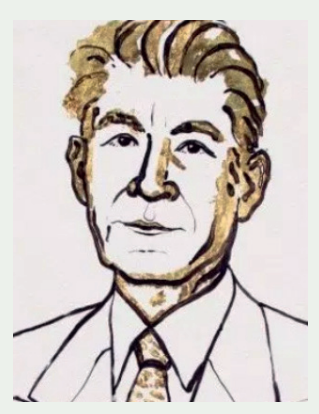

Tasuku Honjo est né en 1942 à Kyoto. II a obtenu son diplôme de médecine en 1966 à I'université de Kyoto. Après avoir fait son internat en 1966-1967 et étudié la chimie médicale de 1967 à 1971, toujours à l'université de Kyoto, il a été post-doc de 1971 à 1974 à Baltimore (grâce à une bourse de recherche de la « Carnegie Institution »), puis au laboratoire de génétique moléculaire du «National Institute of Child Health and Human development » des NIH à Bethesda (Maryland) et a obtenu son doctorat en sciences (PhD) à I'université de Kyoto en 1975. Il a été alors « assistant professor » (équivalent de maître de conférences) à l'université de Tokyo de 1974 à 1979 puis à l'université d'Osaka de 1979 à 1984.

Il est professeur dans le département d'immunologie et de médecine génomique de la Faculté de médecine de l'université de Kyoto depuis 1984, faculté dont il a été le doyen de 1996 à 2000 puis de 2002 à 2004. Parmi ses nombreuses autres fonctions, il a participé au comité éditorial des Annales de l'Institut Pasteur de 1986 à 1988. Ses études sur la molécule PD-1 et ses fonctions ont été menées à l'université de Kyoto, notamment celles portant sur le rôle inhibiteur de PD-l sur la réponse anti-tumorale qu'il a démontré en utilisant un anticorps dirigé contre le ligand de PD-1, PD-L1, bloquant l'interaction PD-1/PD-L1, ainsi que des souris dont le gène codant PD-1 avait été invalidé [2]. médecine/sciences, devant ces succès majeurs de l'immunothérapie des cancers auxquels James Allison et Tasuku Honjo ont largement contribué et pour lesquels ils ont été récompensés par l'attribution du prix Nobel précité, a dialogué avec le Professeur Wolf-Hervé Fridman dont le laboratoire a fait des contributions majeures à ce champ, l'immunothérapie des cancers, et aux découvertes fondamentales qui la sous-tendent $(\rightarrow)$. $\diamond$

Cancer immunotherapy crowned with

$(\rightarrow)$ Voir page 367 Nobel Prize in Physiology or Medicine awarded to James Allison and Tasuku Honjo

\section{LIENS D'INTÉRÊT}

L'auteur déclare n'avoir aucun lien d'intérêt concernant les données publiées dans cet article.

\section{RéFÉRENCES}

1. Leach DR, Krummel MF, Allison JP. Enhancement of antitumor immunity by CTLA-4 blockade. Science $1996 ; 271: 1734-6$.

2. Iwai Y, Ishida Y, Tanaka Y, et al. Involvement of PD-Ll on tumor cells in the escape from host immune system and tumor immunotherapy by PD-L1 blockade. Proc Natl Acad Sci USA 2002 ; 99 : 12293-7.

\section{TIRÉS À PART}

J.L. Teillaud
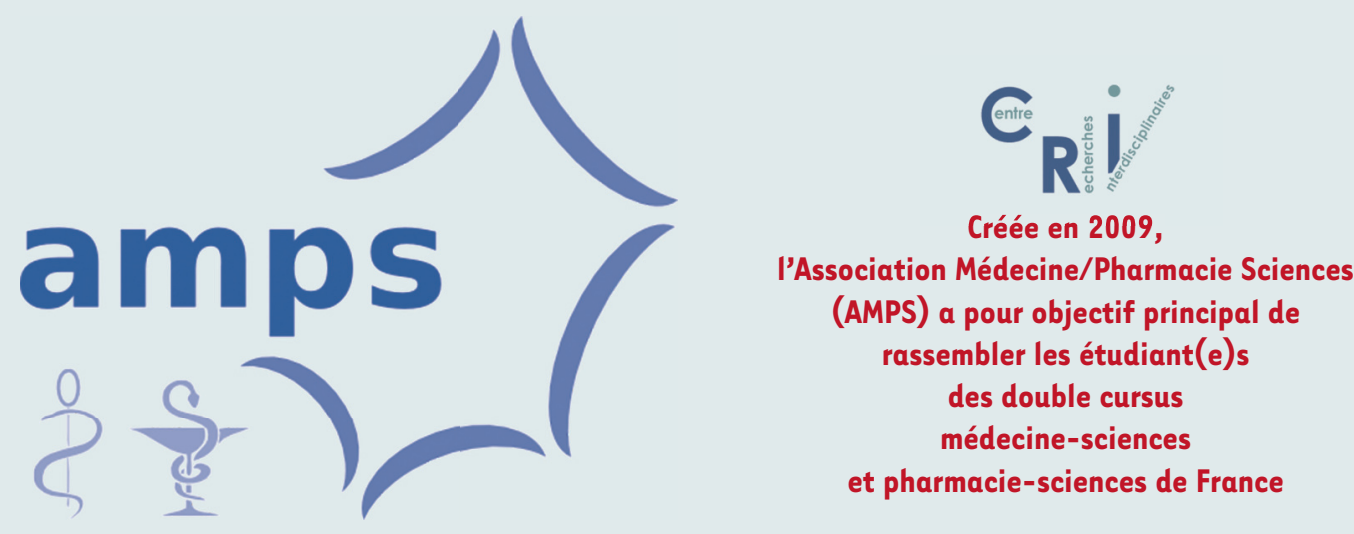

L'AMPS encourage les approches multidisciplinaires et permet aux étudiants des différentes facultés, ayant des compétences différentes, d'échanger leurs idées et d'interagir entre eux, via un groupe virtuel

(sur les réseaux sociaux) performant, des dîners doubles cursus mensuels et un congrès annuel.

Nous comptons parmi nos membres des étudiants en master, des doctorants, des internes et des cliniciens. Cette formidable diversité permet de mettre en commun les différentes expertises scientifiques et cliniques.

Elle permet également aux plus jeunes de bénéficier des conseils précieux de leurs aînés.

La newsletter, envoyée à tous les membres chaque mois, est un outil que chacun utilise au mieux.

http://www.amps-asso.fr

\author{
Groupe facebook: AMPS (Association Médecine Pharmacie Sciences) \\ Sur Twitter : @AssoAMPS
}

